International Journal of Bifurcation and Chaos, Vol. 11, No. 2 (2001) 435-447

(c) World Scientific Publishing Company

\title{
CHAOTIC BEHAVIOR OF THREE COMPETING SPECIES OF MAY-LEONARD MODEL UNDER SMALL PERIODIC PERTURBATIONS*
}

\author{
VALENTIN S. AFRAIMOVICH \\ IICO-UASLP, A.Obregon 64, San Luis Potosi, SLP78000, Mexico \\ SZE-BI HSU ${ }^{\dagger}$ and HUEY-ER LIN \\ Department of Mathematics, National Tsing-Hua University, \\ Hsinchu, Taiwan R.O.C.
}

Received November 5, 1999; Revised April 10, 2000

\begin{abstract}
The influence of periodic perturbations to a Lotka-Volterra system, modeling a competition between three species, is studied, provided that in the unperturbed case the system has a unique attractor - a contour of heteroclinic orbits joining unstable equilibria. It is shown that the perturbed system may manifest regular behavior corresponding to the existence of a smooth invariant torus, and, as well, may have chaotic regimes depending on some parameters. Theoretical results are confirmed by numerical simulations.
\end{abstract}

\section{Introduction}

In this paper we study the asymptotic behavior of the solution for the following periodically perturbed asymmetric May-Leonard system

$$
\left\{\begin{array}{l}
\dot{x_{1}}=x_{1}\left(1-x_{1}-\alpha_{1} x_{2}-\beta_{1} x_{3}\right)+\varepsilon \varphi_{1}(\theta), \\
\dot{x_{2}}=x_{2}\left(1-\beta_{2} x_{1}-x_{2}-\alpha_{2} x_{3}\right)+\varepsilon \varphi_{2}(\theta), \\
\dot{x_{3}}=x_{3}\left(1-\alpha_{3} x_{1}-\beta_{3} x_{2}-x_{3}\right)+\varepsilon \varphi_{3}(\theta), \\
\dot{\theta}=1, \\
x_{1}(0)>0, x_{2}(0)>0, x_{3}(0)>0, \\
\quad \theta(0)=0,0<\varepsilon \ll 1 .
\end{array}\right.
$$

We shall discuss $(1)_{\varepsilon}$ under the assumption

$$
0<\alpha_{i}<1<\beta_{i}, \quad i=1,2,3 .
$$

For $\varepsilon=0$, the Lotka-Volterra system $(1)_{\varepsilon} \bmod -$ els the competition between three species with the same intrinsic growth rates and different competition coefficients [Chi et al., 1998; May, 1975]. From the results of a two-dimensional competitive system [Waltman, 1983], the assumption in (2) ensures that there is an orbit $O_{3}$ on the $x_{1} x_{2}$ plane connecting the equilibrium $e_{2}$ to the equilibrium $e_{1}$, an orbit $O_{2}$ on the $x_{1} x_{3}$ plane connecting the equilibrium $e_{1}$ to the equilibrium $e_{3}$, and an orbit $O_{1}$ on the $x_{2} x_{3}$ plane connecting the equilibrium $e_{3}$ to the equilibrium $e_{2}$, where $e_{1}=(1,0,0), e_{2}=(0,1,0)$ and $e_{3}=(0,0,1)$. In [Chi et al., 1998], the authors proved the global asympototic behavior of the solutions for the unperturbed system $(1)_{\varepsilon}, \varepsilon=0$, as follows: under the assumption (2), the unperturbed system has a unique positive interior equilibrium $P=\left(p_{1}, p_{2}, p_{3}\right)$ and $P$ is globally asymptotically stable provided $\nu_{11} \nu_{21} \nu_{31}<1$, while $P$ is a saddle point with one-dimensional stable manifold $\Gamma$ provided $\nu_{11} \nu_{21} \nu_{31}>1$ where $\nu_{i 1}=\left(\beta_{i}-1\right) /\left(1-\alpha_{i}\right)$, $i=1,2,3$. There exists no periodic solutions for the case $\nu_{11} \nu_{21} \nu_{31} \neq 1$. If $\nu_{11} \nu_{21} \nu_{31}>1$, then the $\omega$-limit set $\omega\left(x_{0}\right)=O_{1} \cup O_{2} \cup O_{3}$ for $x_{0} \notin \Gamma$. For

\footnotetext{
*The work was done while the first author visited National Center of Theoretical Science, Hsinchu, Taiwan in April 1999.
}

${ }^{\dagger}$ Partially supported by National Council of Science, R.O.C. 
the case $\nu_{11} \nu_{21} \nu_{31}=1$, the degenerate Hopf bifurcation occurs and there is a family of neutrally stable periodic solutions.

Under the assumption (2) and $\nu_{11} \nu_{21} \nu_{31}>1$, in Sec. 2 we construct local and global maps to derive a model map for the periodically perturbed system $(1)_{\varepsilon}$. We shall analyze the model map and study the behavior of the iterates of the model map in certain parameter range in Sec. 3. When the parameter is sufficiently small, we prove that the solution of the model map is quite regular by annulus principle [Afraimovich et al., 1983]. For the relatively large parameter, we show that the model map is topologically conjugate to the Bernoulli shift with two symbols by constructing a geometric Smale horseshoe and checking the hyperbolic conditions for the geometric Smale horseshoe.

\section{Derivation of the Model Map}

Consider the asymmetric May-Leonard system [Chi et al., 1998]

$$
\left\{\begin{array}{l}
\dot{x_{1}}=x_{1}\left(1-x_{1}-\alpha_{1} x_{2}-\beta_{1} x_{3}\right) \\
\dot{x_{2}}=x_{2}\left(1-\beta_{2} x_{1}-x_{2}-\alpha_{2} x_{3}\right) \\
\dot{x_{3}}=x_{3}\left(1-\alpha_{3} x_{1}-\beta_{3} x_{2}-x_{3}\right) \\
x_{1}(0)>0, x_{2}(0)>0, x_{3}(0)>0
\end{array}\right.
$$

where

$$
0<\alpha_{i}<1<\beta_{i}<2, \quad i=1,2,3,
$$

and its perturbed system

$$
\left\{\begin{array}{l}
\dot{x_{1}}=x_{1}\left(1-x_{1}-\alpha_{1} x_{2}-\beta_{1} x_{3}\right)+\varepsilon \varphi_{1}(\theta) \\
\dot{x_{2}}=x_{2}\left(1-\beta_{2} x_{1}-x_{2}-\alpha_{2} x_{3}\right)+\varepsilon \varphi_{2}(\theta) \\
\dot{x_{3}}=x_{3}\left(1-\alpha_{3} x_{1}-\beta_{3} x_{2}-x_{3}\right)+\varepsilon \varphi_{3}(\theta) \\
\dot{\theta}=1 \\
x_{1}(0)>0, x_{2}(0)>0, x_{3}(0)>0, \theta(0)=0
\end{array}\right.
$$

where $\varphi_{i}$ are smooth, positive and periodic with period $2 \pi$, and $0<\varepsilon \ll 1$. We note that the basic assumption (H1) is a special case of (2) and (H1) will specify some "leading" directions as we see later. We are interested in the behavior of the solutions for the system (4). Before studying it, some results about the system (3) which can be found in [Chi et al., 1998] are stated as follows. There are equilibria points $e_{1}=(1,0,0), e_{2}=$ $(0,1,0), e_{3}=(0,0,1)$ and $P=\left(p_{1}, p_{2}, p_{3}\right)$ with $p_{i}>0$ for (3). Let $V(x)$ be the variational matrix of the system (3). We have the following tabulated results:

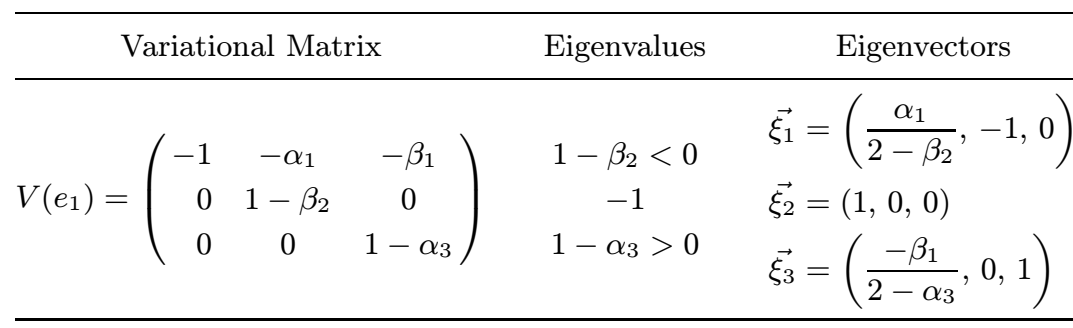

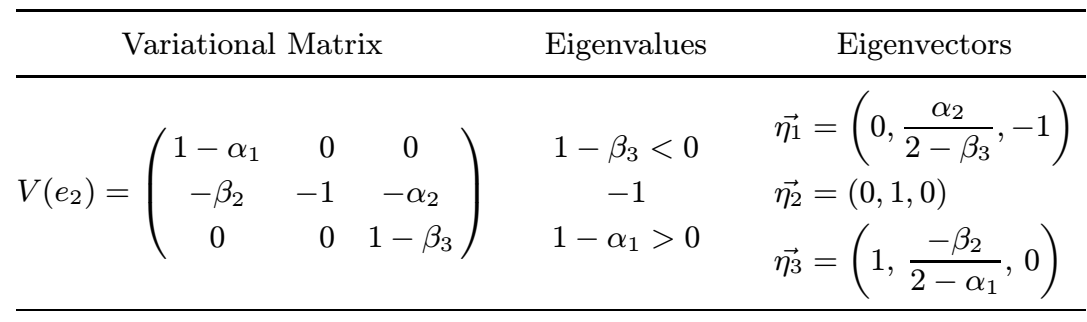

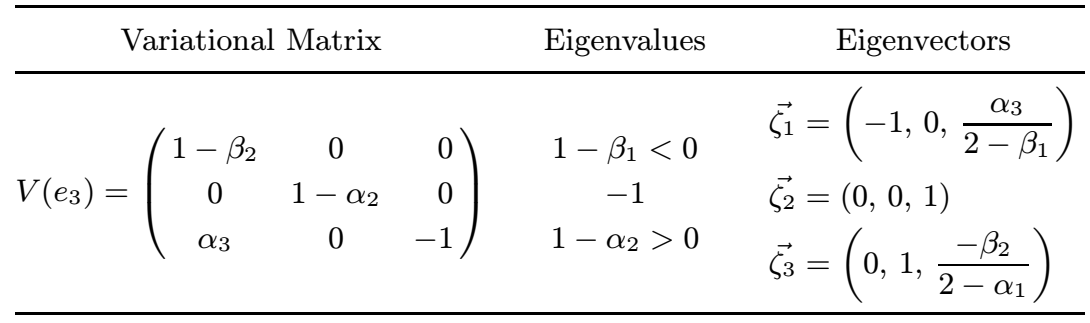




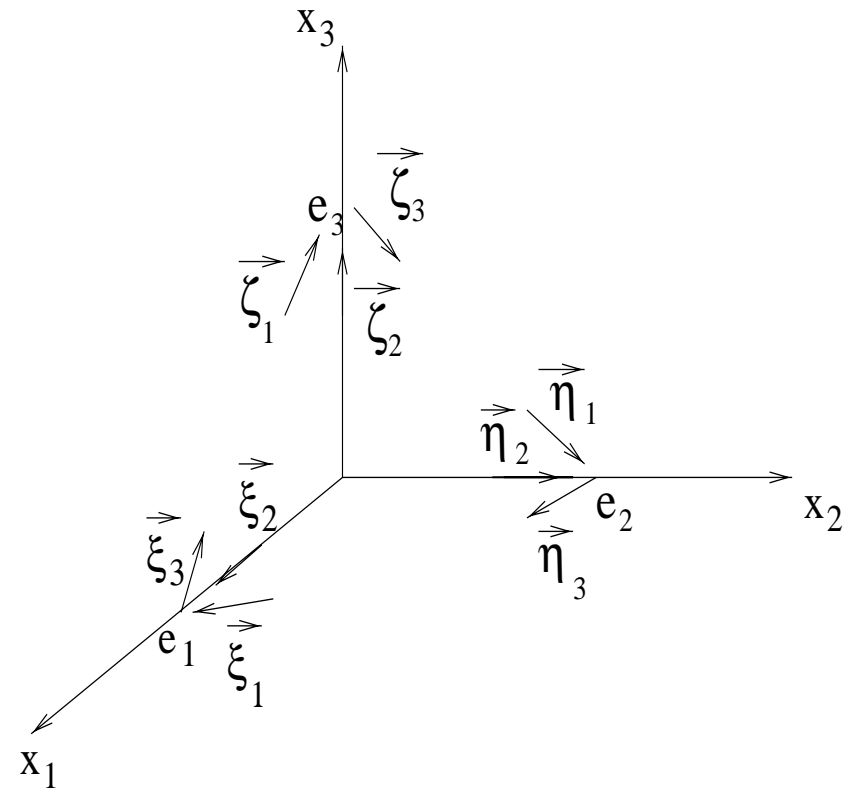

Fig. 1. Eigenvectors of variational matrices $V\left(e_{i}\right), i=$ $1,2,3$.

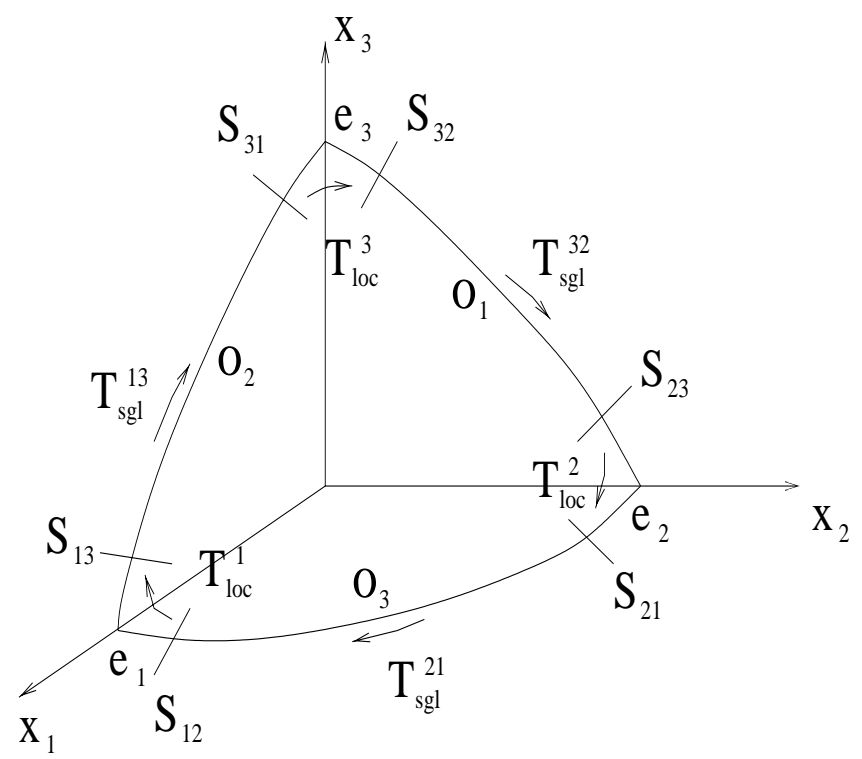

Fig. 2. Constructions of transversal sections for local maps and global maps.

Obviously, $E_{1}^{s}=\left\{t_{1} \vec{\xi}_{1}+t_{2} \vec{\xi}_{2} \mid t_{1}, t_{2} \in \mathbf{R}\right\}$ and $E_{1}^{u}=$ $\left\{t \vec{\xi}_{3} \mid t \in \mathbf{R}\right\}$ are the stable and unstable manifolds of the linearized system $\vec{x}^{\prime}=V\left(e_{1}\right) \vec{x}$, respectively. From $(\mathbf{H 1})$, we have $-1<1-\beta_{2}<0$, and hence $\overrightarrow{\xi_{1}}$ corresponds to the leading direction for solutions of (3) which is asymptotic to $e_{1}$ in $x_{1} x_{2}$ plane as $t \rightarrow$ $\infty$ (see Fig. 1). Similarly, $E_{2}^{s}=\left\{t_{1} \vec{\eta}_{1}+t_{2} \vec{\eta}_{2} \mid t_{1}, t_{2} \in\right.$ $\mathbf{R}\}$ and $E_{2}^{u}=\left\{t \vec{\eta}_{3} \mid t \in \mathbf{R}\right\}$ are the stable and unstable manifolds of the linearized system $\vec{x}^{\prime}=V\left(e_{2}\right) \vec{x}$, respectively. $E_{3}^{s}=\left\{t_{1} \vec{\zeta}_{1}+t_{2} \vec{\zeta}_{2} \mid t_{1}, t_{2} \in \mathbf{R}\right\}$ and $E_{3}^{u}=\left\{t \vec{\zeta}_{3} \mid t \in \mathbf{R}\right\}$ are the stable and unstable manifolds of the linearized system $\vec{x}^{\prime}=V\left(e_{3}\right) \vec{x}$, respectively. $\vec{\eta}_{1}$ and $\vec{\zeta}_{1}$ are the corresponding leading directions of orbits for the system (3) which approach $e_{2}$ and $e_{3}$ as $t \rightarrow \infty$, respectively. Set $\lambda_{j 1}=1-\beta_{j}$, $\lambda_{j 2}=-1, \lambda_{j 3}=1-\alpha_{j}, \nu_{j 1}=-\left(\lambda_{j 1} / \lambda_{j 3}\right)$ and $\nu_{j 2}=-\left(\lambda_{j 2} / \lambda_{j 3}\right)$ for $j=1,2,3$. $P$ is global asymptotically stable if $\nu_{11} \nu_{21} \nu_{31}<1$ and it is a saddle point with one-dimensional stable manifold $\Gamma$ if $\nu_{11} \nu_{21} \nu_{31}>1$. Furthermore, if $x_{0} \notin \Gamma$, then the omega limit set $\omega\left(x_{0}\right)=O_{1} \cup O_{2} \cup O_{3}$, where $O_{1}$ is an orbit connecting $e_{3}$ and $e_{2}, O_{3}$ is an orbit connecting $e_{2}$ and $e_{1}$, and $O_{2}$ is an orbit connecting $e_{1}$ and $e_{3}$ (see Fig. 2).

In this paper our second basic assumption is

$$
\nu:=\nu_{11} \nu_{21} \nu_{31}>1 \text {. }
$$

In the following we construct the Poincare map as a composition of local maps and global maps for system (3). Introduce a new coordinate $\left(\xi_{1}, \xi_{2}, \xi_{3}\right)$ in the neighborhood of $e_{1}$. Then, system (3) in a small neighborhood of $e_{1}$ can be written in the form

$$
\left\{\begin{array}{l}
\dot{\xi_{1}}=\lambda_{11} \xi_{1}+\cdots \\
\dot{\xi_{2}}=\lambda_{12} \xi_{2}+\cdots \\
\dot{\xi_{3}}=\lambda_{13} \xi_{3}+\cdots
\end{array}\right.
$$

Let us choose two sections $S_{12}$ and $S_{13}$ tranversal to the flow

$$
\begin{aligned}
& S_{12}=\left\{\left(\xi_{1}, \xi_{2}, \xi_{3}\right): \xi_{1}=d_{11}, \xi_{2}^{2}+\xi_{3}^{2} \leq d_{12}\right\} \\
& S_{13}=\left\{\left(\xi_{1}, \xi_{2}, \xi_{3}\right): \xi_{3}=d_{13}, \xi_{1}^{2}+\xi_{2}^{2} \leq d_{12}\right\} .
\end{aligned}
$$

Let $\left(d_{11}, \xi_{20}, \xi_{30}\right) \in S_{12}$. The orbit of (5) through $\left(d_{11}, \xi_{20}, \xi_{30}\right)$ is

$$
\left\{\begin{array}{l}
\xi_{1}(t)=\exp \left(\lambda_{11} t\right) d_{11}+\cdots, \\
\xi_{2}(t)=\exp \left(\lambda_{12} t\right) \xi_{20}+\cdots, \\
\xi_{3}(t)=\exp \left(\lambda_{13} t\right) \xi_{30}+\cdots
\end{array}\right.
$$

Obviously, the transition time from $S_{12}$ to $S_{13}$, denoted by $t_{1}$, satisfies $\xi_{3}\left(t_{1}\right)=d_{13}$. Then $t_{1} \approx$ $\left(1 / \lambda_{13}\right) \ln \left(d_{13} / \xi_{30}\right)$, and

$$
\begin{aligned}
& \xi_{1}\left(t_{1}\right)=: \bar{\xi}_{1}=d_{11}\left(\frac{\xi_{30}}{d_{13}}\right)^{\nu_{11}}, \\
& \xi_{2}\left(t_{1}\right)=: \bar{\xi}_{2}=\xi_{30}\left(\frac{\xi_{20}}{d_{13}}\right)^{\nu_{12}} .
\end{aligned}
$$


Hence, the local map $T_{\text {loc }}^{1}: S_{12} \rightarrow S_{13}, T_{\text {loc }}^{1}\left(\xi_{20}\right.$, $\left.\xi_{30}\right)=\left(\bar{\xi}_{1}, \bar{\xi}_{2}\right)$ satisfies

$$
\left\{\begin{array}{l}
\bar{\xi}_{1}=A_{11} \xi_{30}^{\nu_{11}}+\cdots, \\
\bar{\xi}_{2}=A_{21} \xi_{30}^{\nu_{12}} \xi_{20}+\cdots,
\end{array}\right.
$$

where $A_{11}=d_{11}\left(d_{13}\right)^{-\nu_{11}}, A_{21}=\left(d_{13}\right)^{-\nu_{12}}, \nu_{11}=$ $-\left(\lambda_{11} / \lambda_{13}\right)>0$ and $\nu_{12}=-\left(\lambda_{12} / \lambda_{13}\right)>0$ (see Fig. 2).

For the system (4), let the sections be

$$
\begin{aligned}
\hat{S}_{12}= & \left\{\left(\xi_{1}, \xi_{2}, \xi_{3}, \theta_{12}\right):\right. \\
& \left.\xi_{1}=d_{11}, \xi_{2}^{2}+\xi_{3}^{2} \leq d_{12}, 0 \leq \theta_{12}<2 \pi\right\}, \\
\hat{S}_{13}= & \left\{\left(\xi_{1}, \xi_{2}, \xi_{3}, \theta_{13}\right):\right. \\
& \left.\xi_{3}=d_{13}, \xi_{1}^{2}+\xi_{2}^{2} \leq d_{12}, 0 \leq \theta_{13}<2 \pi\right\} .
\end{aligned}
$$

Then, the local map $\hat{T}_{\text {loc }}^{1}:\left(\xi_{20}, \xi_{30}, \theta_{12}\right) \rightarrow\left(\bar{\xi}_{1}, \bar{\xi}_{2}\right.$, $\left.\theta_{13}\right)$ from $\hat{S}_{12}$ to $\hat{S}_{13}$ is defined as

$$
\left\{\begin{array}{l}
\bar{\xi}_{1}=A_{11} \xi_{30}^{\nu_{11}}+\cdots \\
\bar{\xi}_{2}=A_{21} \xi_{30}^{\nu_{12}} \xi_{20}+\cdots \\
\theta_{13}=\theta_{12}+\frac{1}{\lambda_{13}} \ln \frac{d_{13}}{\xi_{30}}+\cdots(\bmod 2 \pi)
\end{array}\right.
$$

In the same way, let us introduce new coordinates $\left(\eta_{1}, \eta_{2}, \eta_{3}\right)$ and $\left(\zeta_{1}, \zeta_{2}, \zeta_{3}\right)$ in the neighborhoods of $e_{2}$ and $e_{3}$, respectively, and choose the transversal sections

$$
\begin{aligned}
S_{23}= & \left\{\left(\eta_{1}, \eta_{2}, \eta_{3}\right): \eta_{1}=d_{21}, \eta_{2}^{2}+\eta_{3}^{2} \leq d_{22}\right\}, \\
S_{21}= & \left.\left(\eta_{1}, \eta_{2}, \eta_{3}\right): \eta_{3}=d_{23}, \eta_{1}^{2}+\eta_{2}^{2} \leq d_{22}\right\}, \\
S_{31}= & \left.\left(\zeta_{1}, \zeta_{2}, \zeta_{3}\right): \zeta_{1}=d_{31}, \zeta_{2}^{2}+\zeta_{3}^{2} \leq d_{32}\right\}, \\
S_{32}= & \left\{\left(\zeta_{1}, \zeta_{2}, \zeta_{3}\right): \zeta_{3}=d_{33}, \zeta_{1}^{2}+\zeta_{2}^{2} \leq d_{32}\right\} . \\
\hat{S}_{23}= & \left\{\left(\eta_{1}, \eta_{2}, \eta_{3}, \theta_{23}\right):\right. \\
& \left.\eta_{1}=d_{21}, \eta_{2}^{2}+\eta_{3}^{2} \leq d_{22}, 0 \leq \theta_{23}<2 \pi\right\}, \\
\hat{S}_{21}= & \left\{\left(\eta_{1}, \eta_{2}, \eta_{3}, \theta_{21}\right):\right. \\
& \left.\eta_{3}=d_{23}, \eta_{1}^{2}+\eta_{2}^{2} \leq d_{22}, 0 \leq \theta_{21}<2 \pi\right\}, \\
\hat{S}_{31}= & \left\{\left(\zeta_{1}, \zeta_{2}, \zeta_{3}, \theta_{31}\right):\right. \\
& \left.\zeta_{1}=d_{31}, \zeta_{2}^{2}+\zeta_{3}^{2} \leq d_{32}, 0 \leq \theta_{31}<2 \pi\right\}, \\
\hat{S}_{32}= & \left\{\left(\zeta_{1}, \zeta_{2}, \zeta_{3}, \theta_{32}\right):\right. \\
& \left.\zeta_{3}=d_{33}, \zeta_{2}^{2}+\zeta_{3}^{2} \leq d_{32}, 0 \leq \theta_{32}<2 \pi\right\} .
\end{aligned}
$$

Then the local maps can be written as follows $T_{\text {loc }}^{2}:\left(\eta_{20}, \eta_{30}\right) \rightarrow\left(\overline{\eta_{1}}, \overline{\eta_{2}}\right)$ from $S_{23}$ to $S_{21}$ is

$$
\left\{\begin{array}{l}
\overline{\eta_{1}}=A_{12} \eta_{30}^{\nu_{21}}+\cdots \\
\overline{\eta_{2}}=A_{22} \eta_{30}^{\nu_{22}} \eta_{20}+\cdots
\end{array}\right.
$$

$\hat{T}_{\text {loc }}^{2}:\left(\eta_{20}, \eta_{30}, \theta_{23}\right) \rightarrow\left(\overline{\eta_{1}}, \overline{\eta_{2}}, \theta_{21}\right)$ from $\hat{S}_{23}$ to $\hat{S}_{21}$ is

$$
\left\{\begin{array}{l}
\overline{\eta_{1}}=A_{12} \eta_{30}^{\nu_{21}}+\cdots \\
\overline{\eta_{2}}=A_{22} \eta_{30}^{\nu_{22}} \eta_{20}+\cdots \\
\theta_{21}=\theta_{23}+\frac{1}{\lambda_{23}} \ln \frac{d_{23}}{\eta_{30}}+\cdots(\bmod 2 \pi)
\end{array}\right.
$$

where $A_{12}=d_{21}\left(d_{23}\right)^{-\nu_{21}}, A_{22}=\left(d_{23}\right)^{-\nu_{22}}, \nu_{21}=$ $-\left(\lambda_{21} / \lambda_{23}\right)>0$ and $\nu_{22}=-\left(\lambda_{22} / \lambda_{23}\right)>0$;

$T_{\mathrm{loc}}^{3}:\left(\zeta_{20}, \zeta_{30}\right) \rightarrow\left(\bar{\zeta}_{1}, \bar{\zeta}_{2}\right)$ from $S_{31}$ to $S_{32}$ is

$$
\left\{\begin{array}{l}
\bar{\zeta}_{1}=A_{13} \zeta_{30}^{\nu_{31}}+\cdots, \\
\bar{\zeta}_{2}=A_{23} \zeta_{30}^{\nu_{32}} \zeta_{20}+\cdots ;
\end{array}\right.
$$

$\hat{T}_{\text {loc }}^{3}:\left(\zeta_{20}, \zeta_{30}, \theta_{31}\right) \rightarrow\left(\bar{\zeta}_{1}, \bar{\zeta}_{2}, \theta_{32}\right)$ from $\hat{S}_{31}$ to $\hat{S}_{32}$ is

$$
\left\{\begin{array}{l}
\bar{\zeta}_{1}=A_{13} \zeta_{30}^{\nu_{31}}+\cdots \\
\bar{\zeta}_{2}=A_{23} \zeta_{30}^{\nu_{32}} \zeta_{20}+\cdots \\
\theta_{32}=\theta_{31}+\frac{1}{\lambda_{33}} \ln \frac{d_{33}}{\zeta_{30}}+\cdots(\bmod 2 \pi)
\end{array}\right.
$$

where $A_{13}=d_{31}\left(d_{33}\right)^{-\nu_{31}}, A_{23}=\left(d_{33}\right)^{-\nu_{32}}, \nu_{31}=$ $-\left(\lambda_{31} / \lambda_{33}\right)>0$ and $\nu_{32}=-\left(\lambda_{32} / \lambda_{33}\right)>0$ (see Fig. 2).

By neglecting the nonleading terms and higher order terms in (8), (10), (12), we have the simplified local maps written as

$$
\begin{gathered}
\hat{T}_{\text {sloc }}^{1}:\left\{\begin{array}{l}
\bar{\xi}_{1}=A_{11} \xi_{30}^{\nu_{11}}, \bar{\xi}_{2}=0, \\
\theta_{13}=\theta_{12}+\frac{1}{\lambda_{13}} \ln \left(\frac{d_{13}}{\xi_{30}}\right)(\bmod 2 \pi) ;
\end{array}\right. \\
\hat{T}_{\text {sloc }}^{2}:\left\{\begin{array}{l}
\bar{\eta}_{1}=A_{12} \eta_{30}^{\nu_{21}}, \overline{\eta_{2}}=0, \\
\theta_{21}=\theta_{23}+\frac{1}{\lambda_{23}} \ln \left(\frac{d_{23}}{\eta_{30}}\right)(\bmod 2 \pi) ;
\end{array}\right.
\end{gathered}
$$




$$
\hat{T}_{\text {sloc }}^{3}:\left\{\begin{array}{l}
\bar{\zeta}_{1}=A_{13} \zeta_{30}^{\nu_{31}}, \bar{\zeta}_{2}=0, \\
\theta_{32}=\theta_{31}+\frac{1}{\lambda_{33}} \ln \left(\frac{d_{33}}{\zeta_{30}}\right)(\bmod 2 \pi) .
\end{array}\right.
$$

For the system (3), we introduce a global map $T_{\mathrm{gl}}^{13}: S_{13} \rightarrow S_{31},\left(\bar{\xi}_{1}, \bar{\xi}_{2}, \theta_{13}\right) \rightarrow\left(\zeta_{20}, \zeta_{30}, \theta_{31}\right)$ in the neighborhood of the orbit $\mathrm{O}_{2}$ (see Fig. 2). The transition time $t_{13}$ from $S_{13}$ to $S_{31}$ is finite. Therefore, the map $T_{\mathrm{gl}}^{13}$ is a diffeomorphism which can be represented as

$$
\left\{\begin{array}{l}
\zeta_{20}=\zeta_{20}^{\star}+a_{11}^{(13)} \bar{\xi}_{1}+a_{12}^{(13)} \bar{\xi}_{2}+\cdots, \\
\zeta_{30}=\zeta_{30}^{\star}+a_{21}^{(13)} \bar{\xi}_{1}+a_{22}^{(13)} \overline{\xi_{2}}+\cdots .
\end{array}\right.
$$

If $x_{2}(0)=0$ in the system (3), then the solution $\vec{x}(t)=\left(x_{1}(t), x_{2}(t), x_{3}(t)\right)$ has a zero component $x_{2}(t)$ for all $t \in \mathbf{R}$ because of the uniqueness of the initial value problem. Hence, $\bar{\xi}_{1}=0$ is mapped into $\zeta_{30}=0$ (see Fig. 1) and then from (16) we have $\zeta_{30}^{\star}=0$ and $a_{22}^{(13)}=0$. These imply $a_{12}^{(13)} \neq 0$ and $a_{21}^{(13)} \neq 0$ since $T_{\mathrm{gl}}^{13}$ is a diffeomorphism. Hence, the global map $T_{\mathrm{gl}}^{13}$ has the form

$$
\left\{\begin{array}{l}
\zeta_{20}=\zeta_{20}^{\star}+a_{11}^{(13)} \bar{\xi}_{1}+a_{12}^{(13)} \bar{\xi}_{2}+\cdots, \\
\zeta_{30}=a_{21}^{(13)} \bar{\xi}_{1}+\cdots
\end{array}\right.
$$

The system (4) is a perturbation of the system (3) and it is reasonable to write $\hat{T}_{\mathrm{gl}}^{13}: \hat{S}_{13} \rightarrow \hat{S}_{31}$, along a neighborhood of the orbit $\mathrm{O}_{2}$ as

$$
\left\{\begin{aligned}
\zeta_{20}= & \zeta_{20}^{\star}+a_{11}^{(13)} \bar{\xi}_{1}+a_{12}^{(13)} \bar{\xi}_{2} \\
& +\varepsilon \eta_{13}\left(\theta_{13}, \bar{\xi}_{1}, \bar{\xi}_{2}\right)+\cdots \\
\zeta_{30}= & a_{21}^{(13)} \bar{\xi}_{1}+\varepsilon \eta_{13}\left(\theta_{13}, \bar{\xi}_{1}, \bar{\xi}_{2}\right)+\cdots \\
\theta_{31}= & \theta_{13}+t_{13}+\varepsilon \psi_{13}\left(\theta_{13}, \bar{\xi}_{1}, \bar{\xi}_{2}\right) \\
& +\cdots(\bmod 2 \pi) .
\end{aligned}\right.
$$

For simplicity, we assume that $\eta_{13}\left(\theta_{13}, \bar{\xi}_{1}, \bar{\xi}_{2}\right)=$ $\eta_{13}\left(\theta_{13}\right), \psi_{13}\left(\theta_{13}, \bar{\xi}_{1}, \bar{\xi}_{2}\right)=\psi_{13}\left(\theta_{13}\right)$ and $\eta_{13}\left(\theta_{13}\right)$, $\psi_{13}\left(\theta_{13}\right)$ are smooth and $2 \pi$-periodic. By neglecting nonleading and higher order terms, the simplified global map $\hat{T}_{\mathrm{sgl}}^{13}:\left(\bar{\xi}_{1}, \theta_{13}\right) \rightarrow\left(\zeta_{30}, \theta_{31}\right)$ can be written as

$$
\left\{\begin{array}{l}
\zeta_{30}=a_{21}^{(13)} \bar{\xi}_{1}+\varepsilon \eta_{13}\left(\theta_{13}\right), \\
\theta_{31}=\theta_{13}+t_{13}+\varepsilon \psi_{13}\left(\theta_{13}\right)(\bmod 2 \pi) .
\end{array}\right.
$$

Similarly, we introduce a global map $T_{\mathrm{gl}}^{32}: S_{32} \rightarrow$ $S_{23},\left(\bar{\zeta}_{1}, \bar{\zeta}_{2}, \theta_{32}\right) \rightarrow\left(\eta_{20}, \eta_{30}, \theta_{23}\right)$ in the neighborhood of the orbit $O_{1}$. The transition time $t_{32}$ from
$S_{32}$ to $S_{23}$ is finite. Therefore, the map $T_{\mathrm{gl}}^{32}$ is a diffeomorphism which can be represented as

$$
\left\{\begin{array}{l}
\eta_{20}=\eta_{20}^{\star}+a_{11}^{(32)} \bar{\zeta}_{1}+a_{12}^{(32)} \bar{\zeta}_{2}+\cdots \\
\eta_{30}=\eta_{30}^{\star}+a_{21}^{(32)} \bar{\zeta}_{1}+a_{22}^{(32)} \bar{\zeta}_{2}+\cdots
\end{array}\right.
$$

If $x_{1}(0)=0$ in the system (3), then the solution $\vec{x}(t)=\left(x_{1}(t), x_{2}(t), x_{3}(t)\right)$ has a zero component $x_{1}(t)$ for all $t \in \mathbf{R}$. Hence, $\bar{\zeta}_{1}=0$ is mapped into $\eta_{30}=0$ (see Fig. 1) and then from (20) we have $\eta_{30}^{\star}=0$ and $a_{22}^{(32)}=0$. These imply $a_{12}^{(32)} \neq 0$ and $a_{21}^{(32)} \neq 0$ since $T_{\mathrm{gl}}^{32}$ is a diffeomorphism. Hence, the global map $T_{\mathrm{gl}}^{32}$ has the form

$$
\left\{\begin{array}{l}
\eta_{20}=\eta_{20}^{\star}+a_{11}^{(32)} \bar{\zeta}_{1}+a_{12}^{(32)} \bar{\zeta}_{2}+\cdots \\
\eta_{30}=a_{21}^{(32)} \bar{\zeta}_{1}+\cdots
\end{array}\right.
$$

It is reasonable to write $\hat{T}_{\text {gl }}^{32}: \hat{S}_{32} \rightarrow \hat{S}_{23}$, along a neighborhood of the orbit $O_{1}$ as

$$
\left\{\begin{aligned}
\eta_{20}= & \eta_{20}^{\star}+a_{11}^{(32)} \bar{\zeta}_{1}+a_{12}^{(32)} \bar{\zeta}_{2} \\
& +\varepsilon \eta_{32}\left(\theta_{32}, \bar{\zeta}_{1}, \bar{\zeta}_{2}\right)+\cdots \\
\eta_{30}= & a_{21}^{(32)} \bar{\zeta}_{1}+\varepsilon \eta_{32}\left(\theta_{32}, \bar{\zeta}_{1}, \bar{\zeta}_{2}\right)+\cdots \\
\theta_{23}= & \theta_{32}+t_{32}+\varepsilon \psi_{32}\left(\theta_{32}, \bar{\zeta}_{1}, \bar{\zeta}_{2}\right) \\
& +\cdots(\bmod 2 \pi)
\end{aligned}\right.
$$

For simplicity, we assume that $\eta_{32}\left(\theta_{32}, \bar{\zeta}_{1}, \bar{\zeta}_{2}\right)=$ $\eta_{32}\left(\theta_{32}\right), \psi_{32}\left(\theta_{32}, \bar{\zeta}_{1}, \bar{\zeta}_{2}\right)=\psi_{32}\left(\theta_{32}\right)$ and $\eta_{32}\left(\theta_{32}\right)$, $\psi_{32}\left(\theta_{32}\right)$ are smooth and $2 \pi$-periodic. By neglecting nonleading and higher order terms, the simplified global map $\hat{T}_{\mathrm{sgl}}^{32}:\left(\bar{\zeta}_{1}, \theta_{32}\right) \rightarrow\left(\eta_{30}, \theta_{23}\right)$ can be written as

$$
\left\{\begin{array}{l}
\eta_{30}=a_{21}^{(32)} \bar{\zeta}_{1}+\varepsilon \eta_{32}\left(\theta_{32}\right), \\
\theta_{23}=\theta_{32}+t_{32}+\varepsilon \psi_{32}\left(\theta_{32}\right)(\bmod 2 \pi) .
\end{array}\right.
$$

In the same way, the simplified global map $\hat{T}_{\text {sgl }}^{21}$ has the form

$$
\hat{T}_{\mathrm{sgl}}^{21}:\left\{\begin{array}{l}
\xi_{30}=a_{21}^{(21)} \bar{\eta}_{1}+\varepsilon \eta_{21}\left(\theta_{21}\right), \\
\theta_{12}=\theta_{21}+t_{21}+\varepsilon \psi_{21}\left(\theta_{21}\right)(\bmod 2 \pi) .
\end{array}\right.
$$

Thus, we can construct a simplified Poincaré map $\hat{T}_{s}:\left(\bar{\zeta}_{1}, \theta_{32}\right) \rightarrow\left(\overline{\bar{\zeta}}_{1}, \bar{\theta}_{32}\right)$ as a composition $\hat{T}_{s}=$ $\hat{T}_{\text {sloc }}^{3} \circ \hat{T}_{\text {sgl }}^{13} \circ \hat{T}_{\text {sloc }}^{1} \circ \hat{T}_{\text {sgl }}^{21} \circ \hat{T}_{\text {sloc }}^{2} \circ \hat{T}_{\text {sgl }}^{32}$ (see Fig. 2) which are detailed as follows. 
(a) $\hat{T}_{\text {sloc }}^{2} \circ \hat{T}_{\text {sgl }}^{32}$

$$
\left\{\begin{array}{l}
\overline{\eta_{1}}=A_{12}\left(a_{21}^{(32)} \bar{\zeta}_{1}+\varepsilon \eta_{32}\left(\theta_{32}\right)\right)^{\nu_{21}} \\
\theta_{21}=\theta_{32}+t_{32}+\varepsilon \psi_{32}\left(\theta_{32}\right)+\frac{1}{\lambda_{23}} \ln \left(\frac{d_{23}}{a_{21}^{(32)} \bar{\zeta}_{1}+\varepsilon \eta_{32}\left(\theta_{32}\right)}\right) \quad(\bmod 2 \pi)
\end{array}\right.
$$

(b) $\hat{T}_{\text {sgl }}^{21} \circ \hat{T}_{\text {sloc }}^{2} \circ \hat{T}_{\text {sgl }}^{32}$

$$
\left\{\begin{array}{l}
\xi_{30}=a_{21}^{(21)} A_{12}\left(a_{21}^{(32)} \bar{\zeta}_{1}+\varepsilon \eta_{32}\left(\theta_{32}\right)\right)^{\nu_{21}}+\varepsilon \eta_{21}\left(\theta_{21}\right) \\
\theta_{12}=\theta_{32}+t_{32}+t_{21}+\varepsilon \psi_{32}\left(\theta_{32}\right)+\varepsilon \psi_{21}\left(\theta_{21}\right)+\frac{1}{\lambda_{23}} \ln \left(\frac{d_{23}}{a_{21}^{(32)} \bar{\zeta}_{1}+\varepsilon \eta_{32}\left(\theta_{32}\right)}\right) \quad(\bmod 2 \pi)
\end{array}\right.
$$

(c) $\hat{T}_{\text {sloc }}^{1} \circ \hat{T}_{\text {sgl }}^{21} \circ \hat{T}_{\text {sloc }}^{2} \circ \hat{T}_{\text {sgl }}^{32}$

$$
\left\{\begin{aligned}
\bar{\xi}_{1}= & A_{11}\left[a_{21}^{(21)} A_{12}\left(a_{21}^{(32)} \bar{\zeta}_{1}+\varepsilon \eta_{32}\left(\theta_{32}\right)\right)^{\nu_{21}}+\varepsilon \eta_{21}\left(\theta_{21}\right)\right]^{\nu_{11}} \\
\theta_{13}= & \theta_{32}+t_{32}+t_{21}+\varepsilon \psi_{32}\left(\theta_{32}\right)+\varepsilon \psi_{21}\left(\theta_{21}\right)+\frac{1}{\lambda_{23}} \ln \left(\frac{d_{23}}{a_{21}^{(32)} \bar{\zeta}_{1}+\varepsilon \eta_{32}\left(\theta_{32}\right)}\right) \\
& +\frac{1}{\lambda_{13}} \ln \left(\frac{d_{13}}{a_{21}^{(21)} A_{12}\left(a_{21}^{(32)} \bar{\zeta}_{1}+\varepsilon \eta_{32}\left(\theta_{32}\right)\right)^{\nu_{21}}+\varepsilon \eta_{21}\left(\theta_{21}\right)}\right)(\bmod 2 \pi)
\end{aligned}\right.
$$

(d) $\hat{T}_{\text {sgl }}^{13} \circ \hat{T}_{\text {sloc }}^{1} \circ \hat{T}_{\text {sgl }}^{21} \circ \hat{T}_{\text {sloc }}^{2} \circ \hat{T}_{\text {sgl }}^{32}$

$$
\left\{\begin{aligned}
\zeta_{30}= & a_{21}^{(13)} A_{11}\left[a_{21}^{(21)} A_{12}\left(a_{21}^{(32)} \bar{\zeta}_{1}+\varepsilon \eta_{32}\left(\theta_{32}\right)\right)^{\nu_{21}}+\varepsilon \eta_{21}\left(\theta_{21}\right)\right]^{\nu_{11}}+\varepsilon \eta_{13}\left(\theta_{13}\right), \\
\theta_{31}= & \theta_{32}+t_{32}+t_{21}+t_{13}+\varepsilon \psi_{32}\left(\theta_{32}\right)+\varepsilon \psi_{21}\left(\theta_{21}\right)+\varepsilon \psi_{13}\left(\theta_{13}\right)+\frac{1}{\lambda_{23}} \ln \left(\frac{d_{23}}{a_{21}^{(32)} \bar{\zeta}_{1}+\varepsilon \eta_{32}\left(\theta_{32}\right)}\right) \\
& +\frac{1}{\lambda_{13}} \ln \left(\frac{d_{13}}{a_{21}^{(21)} A_{12}\left(a_{21}^{(32)} \bar{\zeta}_{1}+\varepsilon \eta_{32}\left(\theta_{32}\right)\right)^{\nu_{21}}+\varepsilon \eta_{21}\left(\theta_{21}\right)}\right)(\bmod 2 \pi),
\end{aligned}\right.
$$

(e) $\hat{T}_{s}=\hat{T}_{\text {sloc }}^{3} \circ \hat{T}_{\mathrm{sgl}}^{13} \circ \hat{T}_{\mathrm{sloc}}^{1} \circ \hat{T}_{\mathrm{sgl}}^{21} \circ \hat{T}_{\mathrm{sloc}}^{2} \circ \hat{T}_{\mathrm{sgl}}^{32}$

$$
\hat{T}_{s}:\left\{\begin{aligned}
\overline{\bar{\zeta}}_{1}= & A_{13}\left\{a_{21}^{(13)} A_{11}\left[a_{21}^{(21)} A_{12}\left(a_{21}^{(32)} \bar{\zeta}_{1}+\varepsilon \eta_{32}\left(\theta_{32}\right)\right)^{\nu_{21}}+\varepsilon \eta_{21}\left(\theta_{21}\right)\right]^{\nu_{11}}+\varepsilon \eta_{13}\left(\theta_{13}\right)\right\}^{\nu_{31}} \\
:= & \hat{f}\left(\bar{\zeta}_{1}, \theta_{32}\right) \\
\theta_{32}= & \theta_{32}+t_{32}+t_{21}+t_{13}+\varepsilon \psi_{32}\left(\theta_{32}\right)+\varepsilon \psi_{21}\left(\theta_{21}\right)+\varepsilon \psi_{13}\left(\theta_{13}\right) \\
& +\frac{1}{\lambda_{23}} \ln \left(\frac{d_{23}}{a_{21}^{(32)} \bar{\zeta}_{1}+\varepsilon \eta_{32}\left(\theta_{32}\right)}\right)+\frac{1}{\lambda_{13}} \ln \left(\frac{d_{13}}{a_{21}^{(21)} A_{12}\left(a_{21}^{(32)} \bar{\zeta}_{1}+\varepsilon \eta_{32}\left(\theta_{32}\right)\right)^{\nu_{21}}+\varepsilon \eta_{21}\left(\theta_{21}\right)}\right) \\
& +\frac{1}{\lambda_{33}} \ln \left(\frac{d_{33}}{a_{21}^{(13)} A_{11}\left[a_{21}^{(21)} A_{12}\left(a_{21}^{(32)} \bar{\zeta}_{1}+\varepsilon \eta_{32}\left(\theta_{32}\right)\right)^{\nu_{21}}+\varepsilon \eta_{21}\left(\theta_{21}\right)\right]^{\nu_{11}}+\varepsilon \eta_{13}\left(\theta_{13}\right)}\right) \quad(\bmod 2 \pi) \\
:= & \theta_{32}+\hat{g}\left(\bar{\zeta}_{1}, \theta_{32}\right) \quad(\bmod 2 \pi),
\end{aligned}\right.
$$


where

$$
\begin{aligned}
\theta_{21}= & \theta_{32}+t_{32}+\varepsilon \psi_{32}\left(\theta_{32}\right)+\frac{1}{\lambda_{23}} \ln \left(\frac{d_{23}}{a_{21}^{(32)} \bar{\zeta}_{1}+\varepsilon \eta_{32}\left(\theta_{32}\right)}\right) \quad(\bmod 2 \pi), \\
\theta_{13}= & \theta_{32}+t_{32}+t_{21}+\varepsilon \psi_{32}\left(\theta_{32}\right)+\varepsilon \psi_{21}\left(\theta_{21}\right)+\frac{1}{\lambda_{23}} \ln \left(\frac{d_{23}}{a_{21}^{(32)} \bar{\zeta}_{1}+\varepsilon \eta_{32}\left(\theta_{32}\right)}\right) \\
& +\frac{1}{\lambda_{13}} \ln \left(\frac{d_{13}}{a_{21}^{(21)} A_{12}\left(a_{21}^{(32)} \bar{\zeta}_{1}+\varepsilon \eta_{32}\left(\theta_{32}\right)\right)^{\nu_{21}}+\varepsilon \eta_{21}\left(\theta_{21}\right)}\right) \quad(\bmod 2 \pi) .
\end{aligned}
$$

\section{Analysis of the Model Map}

Replace $\bar{\zeta}_{1}, \bar{\zeta}_{1}, \theta_{32}$ and $\overline{\theta_{32}}$ in $(25)$ and $(26)$ by $x, \bar{x}, \theta$, and $\bar{\theta}$, respectively. For the sake of definiteness, let us take $\eta_{32}(\theta)=1+a \sin \theta, \eta_{13}(\theta)=1+b \sin \theta$ and $\eta_{21}(\theta)=1+c \sin \theta$ with $0<a, b, c<1$ for $\hat{T}_{s}$. Let

$$
D=\left\{(x, \theta) \mid \frac{1}{2} A \varepsilon^{\nu}(1-a)^{\nu} \leq x \leq 2 A \varepsilon^{\nu}(1+a)^{\nu}, \quad 0 \leq \theta<2 \pi\right\}
$$

where $A=A_{13}\left(a_{21}^{(13)} A_{11}\right)^{\nu_{31}}\left(a_{21}^{(21)} A_{12}\right)^{\nu_{11} \nu_{31}}$ and $\nu:=\nu_{11} \nu_{21} \nu_{31}>1$.

Lemma 3.1. If $\nu_{21}<1, \nu_{11} \nu_{21}<1$ and $0<\varepsilon \ll 1$, then $D$ is an invariant set for the model map $\hat{T}_{s}$.

Proof. For $(x, \theta) \in D$, since $\varepsilon \ll 1$, we have

$$
\begin{aligned}
\bar{x} & =A_{13}\left\{a_{21}^{(13)} A_{11}\left[a_{21}^{(21)} A_{12}\left(a_{21}^{(32)} x+\varepsilon \eta_{32}(\theta)\right)^{\nu_{21}}+\varepsilon \eta_{21}\left(\theta_{21}\right)\right]^{\nu_{11}}+\varepsilon \eta_{13}\left(\theta_{13}\right)\right\}^{\nu_{31}} \\
& >A_{13}\left\{a_{21}^{(13)} A_{11}\left[a_{21}^{(21)} A_{12}\left(a_{21}^{(32)} x+\varepsilon \eta_{32}(\theta)\right)^{\nu_{21}}\right]^{\nu_{11}}+\varepsilon \eta_{13}\left(\theta_{13}\right)\right\}^{\nu_{31}} \\
& >A_{13}\left(a_{21}^{(13)} A_{11}\right)^{\nu_{31}}\left(a_{(21)}^{21} A_{12}\right)^{\nu_{11} \nu_{31}} \varepsilon^{\nu}(1-a)^{\nu} \\
& =A \varepsilon^{\nu}(1-a)^{\nu} .
\end{aligned}
$$

For $x=O\left(\varepsilon^{\nu}\right), \varepsilon \ll 1, \nu>1$ and $\nu_{21}<1, \nu_{11} \nu_{21}<1, \nu_{31}>1$, we can choose $r_{1}, r_{2}, r_{3}>1$ such that $r_{1}^{\nu_{11} \nu_{21}} r_{2}^{\nu_{31}} r_{3}<2$ and

$$
\begin{aligned}
\bar{x} & <A_{13}\left\{a_{21}^{(13)} A_{11}\left[a_{21}^{(21)} A_{12} r_{1}(\varepsilon(1+a))^{\nu_{21}}+\varepsilon \eta_{21}\left(\theta_{21}\right)\right]^{\nu_{11}}+\varepsilon \eta_{13}\left(\theta_{13}\right)\right\}^{\nu_{31}} \\
& <A_{13}\left\{a_{21}^{(13)} A_{11} r_{2}\left[\left(a_{21}^{(21)} A_{12} r_{1}\right)^{\nu_{11}}(\varepsilon(1+a))^{\nu_{11} \nu_{21}}\right]+\varepsilon \eta_{13}\left(\theta_{13}\right)\right\}^{\nu_{31}} \\
& <A_{13} r_{3}\left(a_{21}^{(13)} A_{11} r_{2}\right)^{\nu_{31}}\left(a_{21}^{(21)} A_{12} r_{1}\right)^{\nu_{11} \nu_{31}} \varepsilon^{\nu}(1+a)^{\nu} \\
& =r_{1}^{\nu_{11} \nu_{31}} r_{2}^{\nu_{31}} r_{3} A_{13}\left(a_{21}^{(13)} A_{11}\right)^{\nu_{31}}\left(a_{21}^{(21)} A_{12}\right)^{\nu_{11} \nu_{31}} \varepsilon^{\nu}(1+a)^{\nu} \\
& <2 A \varepsilon^{\nu}(1+a)^{\nu}
\end{aligned}
$$

hold.

Hence $D$ is an invariant set for the model map $\hat{T}_{s}$. 
Lemma 3.1 also implies that the model map $\hat{T}_{s}$ has an attractor in $D$. Since $\varepsilon \ll 1$, we may neglect the higher order terms of $\varepsilon$ for $\hat{T}_{s}$ under the assumptions of Lemma 3.1. Then the model map $\hat{T}_{s}$ in $(25),(26)$ can be regarded as a perturbation of the following reduced map $F$

$F:\left\{\begin{array}{l}\bar{x}=A(B x+\varepsilon(1+a \sin \theta))^{\nu}:=f(x, \theta), \\ \bar{\theta}=\theta+\tilde{\omega}-\eta \ln (B x+\varepsilon(1+a \sin \theta)) \\ :=\theta+g(x, \theta) \quad(\bmod 2 \pi),\end{array}\right.$

where

$$
\begin{aligned}
A= & A_{13}\left(a_{21}^{(13)} A_{11}\right)^{\nu_{31}}\left(a_{21}^{(21)} A_{12}\right)^{\nu_{11} \nu_{31}}, \\
B= & a_{21}^{(32)}, \eta=\left(\frac{1}{\lambda_{23}}+\frac{\nu_{21}}{\lambda_{13}}+\frac{\nu_{11} \nu_{21}}{\lambda_{33}}\right), \\
\tilde{\omega}= & t_{32}+t_{21}+t_{13}+\frac{1}{\lambda_{23}} \ln d_{23}+\frac{1}{\lambda_{13}} \ln \left(\frac{d_{13}}{a_{21}^{(21)} A_{12}}\right) \\
& +\frac{1}{\lambda_{33}} \ln \left(\frac{d_{33}}{a_{21}^{(13)} A_{11}\left(a_{21}^{(21)} A_{12}\right)^{\nu_{11}}}\right) .
\end{aligned}
$$

The map $F$ is, in fact, "the dissipative separatrix map [Afraimovich \& Hsu, 1998]". Obviously, D is also an invariant set for $F$. Consider the map $F$ restricted on $D$. Then $F$ is a diffeomorphism from $D$ onto its image. The sufficient conditions under which $F$ has a regular behavior will be given.

Theorem 3.2. If $\nu>1, \varepsilon \ll 1$ and $0<a<$ $\left(1 / \sqrt{1+\eta^{2}}\right)$, then there is an invariant closed curve as the maximal attractor for $F$.

To prove Theorem 3.2, we apply the following "Annulus Principle".

Proposition 3.3. ("Annulus Principle [Afraimovich et al., 1985; Afraimovich $6 \mathrm{H} H \mathrm{Hu}, 1998] ")$. Let $T$ : $(x, \theta) \rightarrow(\bar{x}, \theta), x \in \mathbf{R}^{n}, \theta \in R^{m}$, be a map of the following form

$$
\bar{x}=f(x, \theta), \quad \bar{\theta}=\theta+g(x, \theta) \quad(\bmod 2 \pi),
$$

where $f, g$ are differentiable functions which are $2 \pi$ periodic in $\theta=\left(\theta_{1}, \ldots, \theta_{m}\right)$. Assume that $T$ maps an "annulus"

$$
D=\left\{(x, \theta):|x| \leq r_{0}\right\}, \quad r_{0}>0
$$

into its interior. Introduce the following norms of vectors or matrices in $D:\|\cdot\|=\sup _{(x, \theta) \in D}|\cdot|$, where $|\cdot|$ is an Euclidean norm. If

(a) $\left\|\left(I+g_{\theta}\right)^{-1}\right\|<\infty$,

(b) $\left\|f_{x}\right\|<1$,

(c) $1-\left\|\left(I+g_{\theta}\right)^{-1}\right\| \cdot\left\|f_{x}\right\|$

$>2 \sqrt{\left\|\left(I+g_{\theta}\right)^{-1}\right\| \cdot\left\|g_{x}\right\| \cdot\left\|\left(I+g_{\theta}\right)^{-1}\right\| \cdot\left\|f_{\theta}\right\|}$,

(d) $1+\left\|\left(I+g_{\theta}\right)^{-1}\right\| \cdot\left\|f_{x}\right\|<2\left\|\left(I+g_{\theta}\right)^{-1}\right\|$,

where $I$ is the identical $m \times m$-matrix and subscripts indicate the differentiation with respect to corresponding variables, then the maximal attractor in $D$ is an invariant $m$-dimensional torus which is the graph of a smooth function $x=h(\theta), h$ is $2 \pi$ periodic in $\theta$.

Proof. See [Afraimovich et al., 1985; Afraimovich \& Hsu, 1998].

Now, let us come back to prove Theorem 3.2.

Proof. From Lemma 3.1, $F(D) \subset D$ where $D$ is defined in (27). We need to check the sufficient conditions of "Annulus Principle". For $(x, \theta) \in D$, we have $x=O\left(\varepsilon^{\nu}\right), \varepsilon \ll 1, \nu>1$. Then

$$
\begin{aligned}
& 1+g_{\theta}=1-\eta \frac{\varepsilon a \cos \theta}{B x+\varepsilon(1+a \sin \theta)} \simeq 1-\eta \frac{a \cos \theta}{1+a \sin \theta}, \\
& \Rightarrow 0<\inf _{(x, \theta) \in D}\left(1+g_{\theta}\right)<1 \quad \text { if } \quad 0<a<\frac{1}{\sqrt{1+\eta^{2}}}, \\
& \Rightarrow c:=\left\|\left(1+g_{\theta}\right)^{-1}\right\|<\infty ; \\
& \text { (ii) } f_{x}=\nu A B(B x+\varepsilon(1+a \sin \theta))^{\nu-1}:=\alpha(\varepsilon) \text {, } \\
& \Rightarrow \alpha(\varepsilon)=O\left(\varepsilon^{\nu-1}\right), \quad \text { hence }\left\|f_{x}\right\|<1 \text {; }
\end{aligned}
$$




$$
\begin{aligned}
& g_{x}=-\eta \frac{B}{B x+\varepsilon(1+a \sin \theta)} \sim O\left(\varepsilon^{-1}\right), \\
& f_{\theta}=\nu A(B x+\varepsilon(1+a \sin \theta))^{\nu-1} \varepsilon a \cos \theta \sim O\left(\varepsilon^{\nu}\right), \\
\Rightarrow & \sqrt{\left\|\left(1+g_{\theta}\right)^{-1}\right\|^{2} \cdot\left\|g_{x}\right\| \cdot\left\|f_{\theta}\right\|} \sim O\left(\varepsilon^{\frac{\nu-1}{2}}\right), \\
& 1-\left\|\left(1+g_{\theta}\right)^{-1}\right\| \cdot\left\|f_{x}\right\| \sim O(1), \\
& 1+\left\|\left(1+g_{\theta}\right)^{-1}\right\| \cdot\left\|f_{x}\right\|=1+c \cdot \alpha(\varepsilon)<2
\end{aligned}
$$

hence, conditions (c) and (d) in Theorem 3.3 are satisfied.

Therefore, the existence of an invariant closed curve for the map $F$ is guaranteed by the Annulus Principle.

Corollary 3.4. If $\nu_{21}<1, \nu_{11} \nu_{21}<1, \nu>1$, and $0<a<\left(1 / \sqrt{1+\eta^{2}}\right)$, then the model map $\hat{T}_{s}$ has an invariant closed curve as the maximal attractor in $D$ for $\varepsilon \ll 1$.

Proof

(i) $1+\hat{g}_{\theta}=1-\frac{1}{\lambda_{23}} \frac{\varepsilon a \cos \theta}{\left(a_{21}^{(32)} x+\varepsilon \eta_{32}(\theta)\right)}-\frac{1}{\lambda_{13}} \frac{a_{21}^{(21)} A_{12} \nu_{21}(*)^{\nu_{21}-1} \cdot \varepsilon a \cos \theta+\varepsilon c \cos \theta_{21} \cdot \frac{\partial \theta_{21}}{\partial \theta}}{a_{21}^{(21)} A_{12}\left[a_{21}^{(32)} x+\varepsilon \eta_{32}(\theta)\right]^{\nu_{21}}+\varepsilon \eta_{21}\left(\theta_{21}\right)}$

$$
\begin{aligned}
& -\frac{1}{\lambda_{33}} \frac{a_{21}^{(13)} A_{11} \nu_{11}(* *)^{\nu_{11}-1}\left(\nu_{21} a_{21}^{(21)} A_{12}(*)^{\nu_{21}-1} \varepsilon a \cos \theta+\varepsilon c \cos \theta_{21} \cdot \frac{\partial \theta_{21}}{\partial \theta}\right)+\varepsilon b \cos \theta_{13} \cdot \frac{\partial \theta_{13}}{\partial \theta}}{a_{21}^{(13)} A_{11}\left[a_{21}^{(21)} A_{12}(*)^{\nu_{21}}+\varepsilon \eta_{21}\left(\theta_{21}\right)\right]^{\nu_{11}}+\varepsilon \eta_{13}\left(\theta_{13}\right)} \\
& +\cdots, \\
\frac{\partial \theta_{21}}{\partial \theta}= & 1+\varepsilon \frac{\partial \psi_{32}}{\partial \theta}-\frac{1}{\lambda_{23}} \frac{\varepsilon a \cos \theta}{a_{21}^{(32)} x+\varepsilon \eta_{32}(\theta)}, \\
\frac{\partial \theta_{13}}{\partial \theta}= & 1+\varepsilon \frac{\partial \psi_{32}}{\partial \theta}+\varepsilon \frac{\partial}{\partial \theta} \psi_{21}\left(\theta_{21}\right)-\frac{1}{\lambda_{23}} \frac{\varepsilon a \cos \theta}{a_{21}^{(32)} x+\varepsilon \eta_{32}(\theta)}-\frac{1}{\lambda_{13}} \frac{a_{21}^{(21)} A_{12}(*)^{\nu_{21}-1} \varepsilon a \cos \theta+\varepsilon b \cos \theta_{21} \cdot \frac{\partial \theta_{21}}{\partial \theta}}{a_{21}^{(21)} A_{12}(*)^{\nu_{21}}+\varepsilon \eta_{21}\left(\theta_{21}\right)}
\end{aligned}
$$

where $*=: a_{21}^{(32)} x+\varepsilon \eta_{32}(\theta), * *=: a_{21}^{(21)}$ $A_{12}(*)^{\nu_{21}}+\varepsilon \eta_{21}\left(\theta_{21}\right)$, and $\cdots$ denotes higher order terms of $\varepsilon$. Under the assumptions, for $(x, \theta) \in D$, since $\varepsilon \ll 1$ we can neglect higher order terms of $\varepsilon$ in (25) and (26), then

$$
\begin{aligned}
\frac{\partial \theta_{21}}{\partial \theta} & \simeq O(1) \\
\frac{\partial \theta_{13}}{\partial \theta} & \simeq O(1), \\
1+\hat{g}_{\theta} & \simeq 1-\eta \frac{\varepsilon a \cos \theta}{a_{21}^{(32)} x+\varepsilon(1+a \sin \theta)} .
\end{aligned}
$$

From straight-forward computation and the same argument as above, we obtain

(ii) $\hat{f}_{x} \simeq \nu A B(B x+\varepsilon(1+a \sin \theta))^{\nu-1}$,

(iii) $\hat{g}_{x} \simeq-\eta \frac{B}{B x+\varepsilon(1+a \sin \theta)}$,

$$
\hat{f}_{\theta} \simeq \nu A(B x+\varepsilon(1+a \sin \theta))^{\nu-1} \varepsilon a \cos \theta .
$$

Hence, the model map $\hat{T}_{s}$ and the reduced map $F$ are $C^{1}$ closed. So the Annulus Principle can be also applied to $\hat{T}_{s}$ same as we did in Theorem 3.2. Hence we complete the proof.

The chaotic behavior for the map $F$ will be characterized as follows. 
Theorem 3.5. If $\nu>1$ and $1>a>$ $\left(\exp ^{\frac{10 \pi}{\eta}}-1\right) /\left(\exp ^{\frac{10 \pi}{\eta}}-(1 / 10)\right)$, then there exists a hyperbolic invariant closed subset $\Lambda \subset D$ such that $\left.F\right|_{\Lambda}$ is topologically conjugate to the Bernoulli shift with two symbols for $\varepsilon \ll 1$.

To prove the hyperbolicity, we apply the following Theorem which gives sufficient conditions of hyperbolicity [Afraimovich et al., 1983].

Theorem 3.6. Let $F: U \rightarrow \mathbf{R}^{m+n}$ be a $C^{1}$ map, where $U$ is an open convex subset of $\mathbf{R}^{m+n}$, such that $F(x, y)=(\bar{x}, \bar{y}), x \in \mathbf{R}^{m}, y \in \mathbf{R}^{n}$, with the form $\bar{x}=f(x, y), \bar{y}=g(x, y)$. If

(a) $\left\|f_{x}\right\|<1$

(b) $\left\|g_{y}^{-1}\right\|<1$,

(c) $1-\left\|f_{x}\right\|\left\|g_{y}^{-1}\right\|>2 \sqrt{\left\|f_{y} \cdot g_{y}^{-1}\right\|\left\|g_{x}\right\|\left\|g_{y}^{-1}\right\|}$,

(d) $\left(1-\left\|f_{x}\right\|\right)\left(1-\left\|g_{y}^{-1}\right\|\right)>\left\|f_{y} \cdot g_{y}^{-1}\right\| \cdot\left\|g_{x}\right\|$,

where $\|\cdot\|=\sup _{(x, y) \in U}|\cdot|$, and subscripts means differentiation with respect to the corresponding coordinates, then any compact invariant set $\Lambda$ in $U$ is hyperbolic.

Proof. See [Afraimovich et al., 1983; Afraimovich \& Hsu, 1998].

Now, let us come back to prove Theorem 3.5.

Proof. Denote the lifting map of $\bar{\theta}$ by $\hat{\theta}$. For $(x, \theta) \in D$, consider

$$
\begin{aligned}
\frac{\partial \hat{\theta}}{\partial \theta} & =\frac{\partial}{\partial \theta}(\theta+g(x, \theta)) \\
& =1-\eta \frac{\varepsilon a \cos \theta}{B x+\varepsilon(1+a \sin \theta)} \simeq 1-\eta \frac{a \cos \theta}{1+a \sin \theta},
\end{aligned}
$$

since $x=O\left(\varepsilon^{\nu}\right), \varepsilon \ll 1$ and $\nu>1$. Hence $\hat{\theta}(x, \theta)$ is an increasing function of $\theta$ for $(\pi / 2)<\theta<(3 \pi / 2)$.

Take $\theta_{0}=\pi+\sin ^{-1}(1 / 10), \pi<\theta_{0}<(3 \pi / 2)$. Then for $0 \leq \delta<(3 \pi / 2)-\theta_{0}$

$$
\begin{gathered}
\hat{\theta}\left(x, \frac{3 \pi}{2}-\delta\right)-\hat{\theta}\left(x, \theta_{0}\right) \\
=\left(\frac{3 \pi}{2}-\delta-\theta_{0}\right)+\eta \ln \frac{B x+\varepsilon\left(1+a \sin \theta_{0}\right)}{B x+\varepsilon\left(1+a \sin \left(\frac{3 \pi}{2}-\delta\right)\right)} \\
\simeq\left(\frac{3 \pi}{2}-\delta-\theta_{0}\right)+\eta \ln \frac{1-\frac{a}{10}}{1+a \sin \left(\frac{3 \pi}{2}-\delta\right)} \text { for } \varepsilon \ll 1 .
\end{gathered}
$$

Define $P:\left[0,(3 \pi / 2)-\theta_{0}\right) \times[0,1) \rightarrow \mathbf{R}$ by

$$
P(\delta, a)=\eta \ln \frac{1-\frac{a}{10}}{1+a \sin \left(\frac{3 \pi}{2}-\delta\right)} .
$$

Then $P(0, a)=\eta \ln \left(1-\frac{a}{10}\right) /(1-a)$. We have

$$
P(0, a)>10 \pi \text { if } a>\frac{\exp ^{\frac{10 \pi}{\eta}}-1}{\exp ^{\frac{10 \pi}{\eta}}-\frac{1}{10}} .
$$

By continuity of $P(\delta, a)$ with respect to $\delta$, there exists $0<\delta_{0}=\delta_{0}(a)<(3 \pi / 2)-\theta_{0}$ such that
$P\left(\delta_{0}, a\right)>10 \pi$ if $a>\left(\exp ^{\frac{10 \pi}{\eta}}-1\right) /\left(\exp ^{\frac{10 \pi}{\eta}}-(1 / 10)\right)$. Hence,

$$
\hat{\theta}\left(x, \frac{3 \pi}{2}-\delta_{0}\right)-\hat{\theta}\left(x, \theta_{0}\right)>10 \pi \text { if } a>\frac{\exp ^{\frac{10 \pi}{\eta}}-1}{\exp ^{\frac{10 \pi}{\eta}}-\frac{1}{10}} .
$$

Then there exists two disjoint subintervals $I_{1}=$ $\left[\theta_{1}, \theta_{2}\right]$ and $I_{2}=\left[\theta_{3}, \theta_{4}\right]$ with $\theta_{0}<\theta_{1}<\theta_{2}<\theta_{3}<$ $\theta_{4}<\frac{3 \pi}{2}-\delta_{0}$ such that for

$$
D_{1}:=\left\{(x, \theta) \mid \frac{1}{2} A \varepsilon^{\nu}(1-a)^{\nu} \leq x \leq 2 A \varepsilon^{\nu}(1+a)^{\nu}, \theta \in I_{1}\right\},
$$

$D_{2}:=\left\{(x, \theta) \mid \frac{1}{2} A \varepsilon^{\nu}(1-a)^{\nu} \leq x \leq 2 A \varepsilon^{\nu}(1+a)^{\nu}, \theta \in I_{2}\right\}$, 


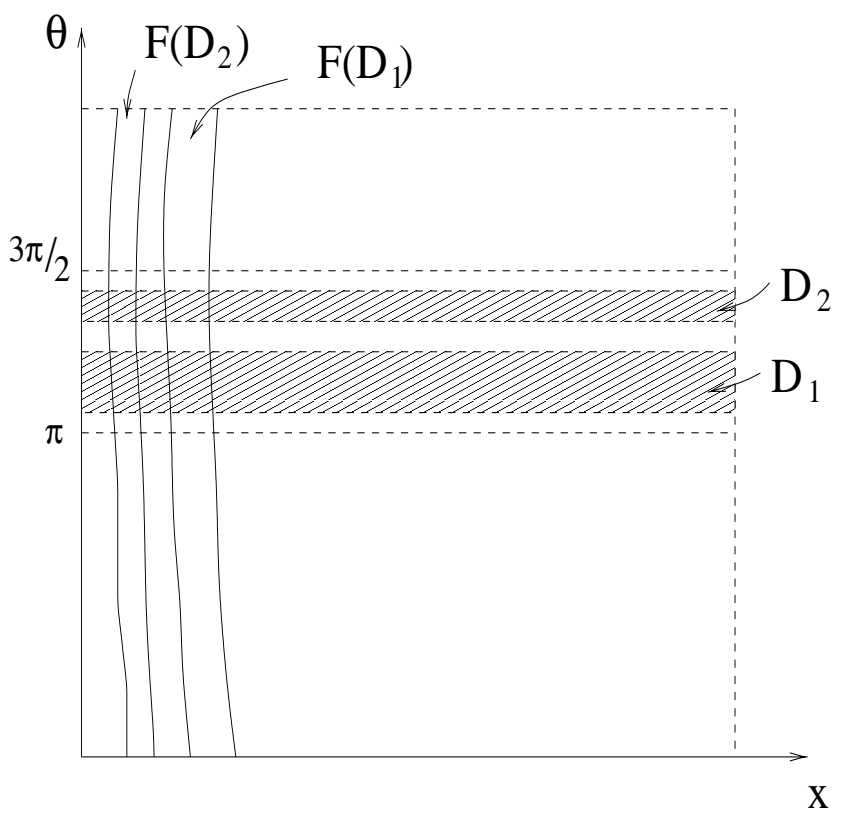

Fig. 3. The images of stripes $D_{1}$ and $D_{2}$ under the map $F$.

we have $F\left(D_{1}\right) \cap F\left(D_{2}\right)=\emptyset$ and both $F\left(D_{1}\right)$ and $F\left(D_{2}\right)$ have full intersections with $D_{1}$ and $D_{2}$ (see Fig. 3). Let $\Lambda=\cap_{-\infty}^{\infty} F^{n}\left(D_{1} \cup D_{2}\right)$. $\Lambda$ is an invariant closed subset of $F$. Thus, we have a "geometric Smale horseshoe" and it can only be said [Burns, 1995] that $\left.F\right|_{\Lambda}$ is topologically semi-conjugate to the Bernoulli shift with two symbols. To achieve our goal, we should check if $\Lambda$ is a hyperbolic set for $F$ by Theorem 3.6. For $\varepsilon \ll 1$ and $\nu>1$, from the calculation in the proof of Theorem 3.2 it follows that:

(i) $\left\|f_{x}\right\|<1$,

(ii) $\left\|\left(1+g_{\theta}\right)^{-1}\right\|<1$ since $\left\|\left(1+g_{\theta}\right)^{-1}\right\|$ $\simeq \sup _{\theta \in I_{1} \cup I_{2}}\left|\left(1-\eta \frac{a \cos \theta}{1+a \sin \theta}\right)^{-1}\right|$

(iii) $\left\|f_{x}\right\|\left\|\left(1+g_{\theta}\right)^{-1}\right\| \sim O\left(\varepsilon^{\nu-1}\right)$,

$\sqrt{\left\|f_{\theta} \cdot\left(1+g_{\theta}\right)^{-1}\right\|\left\|g_{x}\right\|\left\|\left(1+g_{\theta}\right)^{-1}\right\|}$

$\sim O\left(\varepsilon^{\frac{\nu-1}{2}}\right)$,

$\Rightarrow$ the condition (c) in Theorem 3.6 holds.

(iv) $\left(1-\left\|f_{x}\right\|\right)\left(1-\left\|\left(1+g_{\theta}\right)^{-1}\right\|\right) \sim O(1)$,

$\left\|f_{\theta} \cdot\left(1+g_{\theta}\right)^{-1}\right\| \cdot\left\|g_{x}\right\| \sim O\left(\varepsilon^{\nu-1}\right)$

$\Rightarrow$ the condition (d) in Theorem 3.6 holds.

Hence, $\Lambda$ is a hyperbolic set. It implies $\left.F\right|_{\Lambda}$ is topologically conjugate to the Bernoulli shift with two symbols.

Corollary 3.7. If $\nu_{21}<1, \nu_{11} \nu_{21}<1, \nu>1$ and $1>a>\left(\exp ^{\frac{10 \pi}{\eta}}-1\right) /\left(\exp ^{\frac{10 \pi}{\eta}}-(1 / 10)\right)$, then there exists a hyperbolic invariant closed subset $\Lambda \subset$
$D$ such that $\left.\hat{T}_{s}\right|_{\Lambda}$ is topologically conjugate to the Bernoulli shift with two symbols for $\varepsilon \ll 1$.

Proof. We have known the model map $\hat{T}_{s}$ and the reduced map $F$ are $C^{1}$ closed from the Corollary 3.4. Therefore, we may apply general results about structural stability of hyperbolic locally maximal sets (see, e.g. [Katok, 1995]) to conclude that the map $\hat{T}_{s}$ has a hyperbolic locally maximal set, as well, and its restriction to this set is conjugate to the Bernoulli shift. We can also prove it directly since the method that constructs the invariant subset and the arguments which show the hyperbolicity are still valid for $\hat{T}_{s}$. Hence, we complete the proof.

\section{Numerical Results}

In Fig. $4, L_{1}$ and $L_{2}$ denote the curves $a=\left(e^{\frac{10 \pi}{\eta}}-\right.$ 1) $/\left(e^{\frac{10 \pi}{\eta}}-0.1\right)$ and $a=\left(1 / \sqrt{1+\eta^{2}}\right)$, respectively, where $0<a<1$ is the amplitude of perturbation and $0<\eta<\infty$ is defined in (28). For the map in (28), the region above $L_{1}$ is a chaotic region for parameters $a$ and $\eta$. That below $L_{2}$ is a regular region where there exists an invariant closed curve as an attractor. The behavior of $F$ is unknown for the parameter range between $L_{1}$ and $L_{2}$.

For the model map $F$ in (28), let $\eta=100$, $A=B=1, \varepsilon=10^{-3}, \nu=2, \tilde{\omega}=1.2$, and we

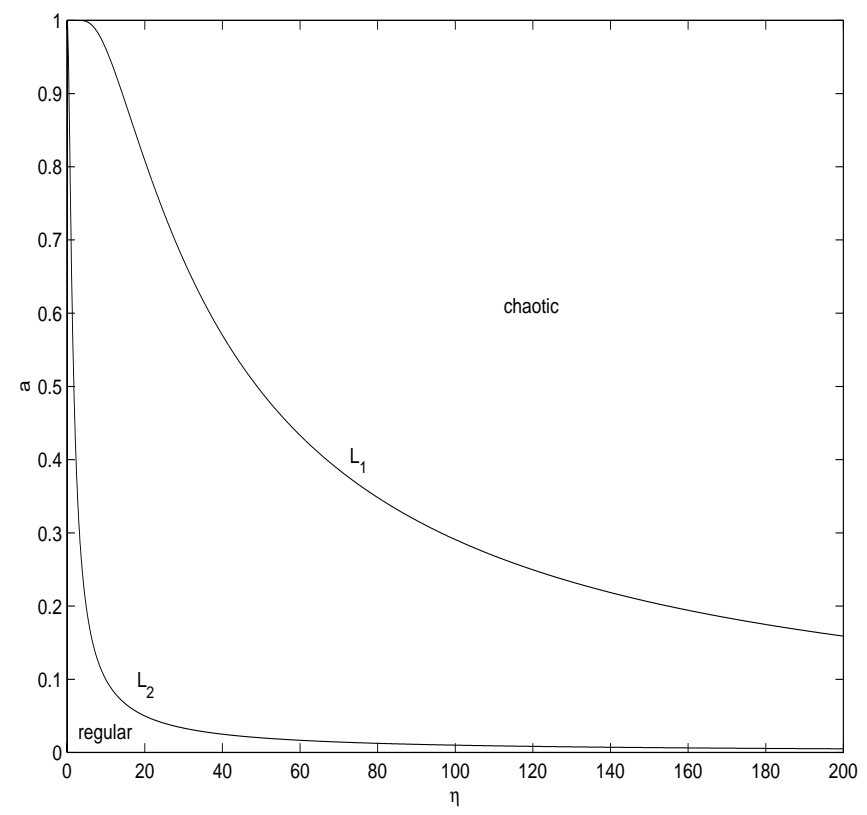

Fig. 4. Regular and chaotic regions of the map $F$ with respect to parameters $a$ and $\eta$. 


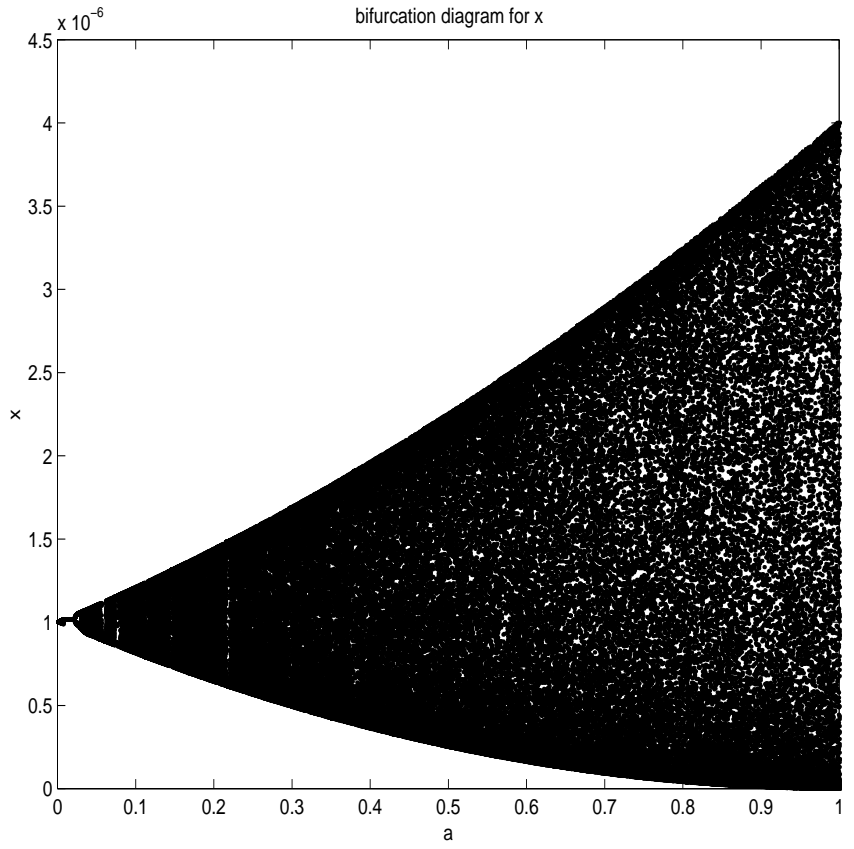

Fig. 5. The bifurcation diagram of $\mathrm{x}$ with respect to the parameter $a$. The last 100 points of 10000 iterations for the map $F$, projected to $x$ axis, are plotted.

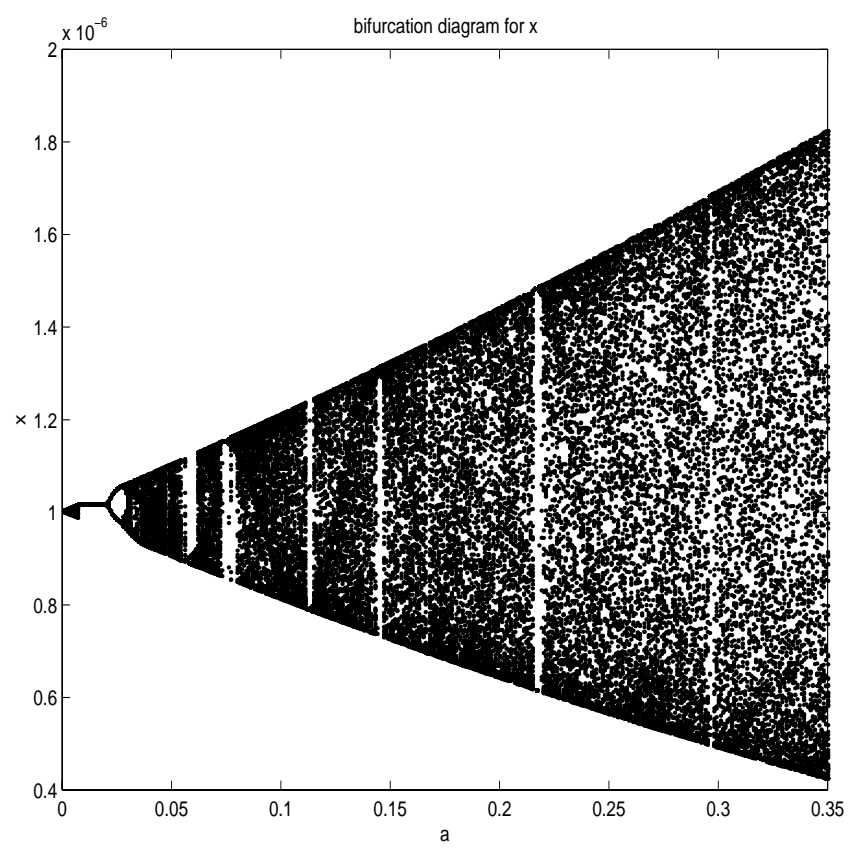

Fig. 6. Part of Fig. 5 restricted to the parameter range $0 \leq a \leq 0.35$.

iterate the map $F 10000$ times with initial datas $x_{1}=10^{-6}, \theta_{1}=1.4 \pi$. The last 100 points are projected to $x$ axis to plot the bifurcation diagrams for $x$ with respect to the parameter $a$ (see Figs. 5 and 6). The last 3000 points are taken to plot the

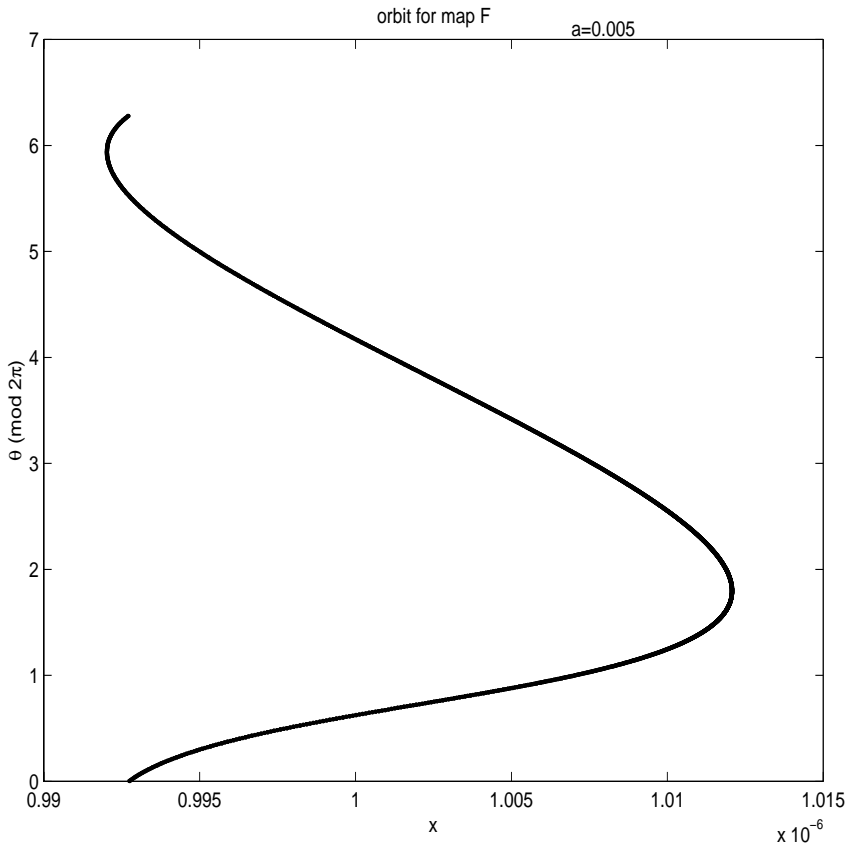

Fig. 7. Orbit of the map $F$ for $a=0.005$ for the last 3000 iterations.

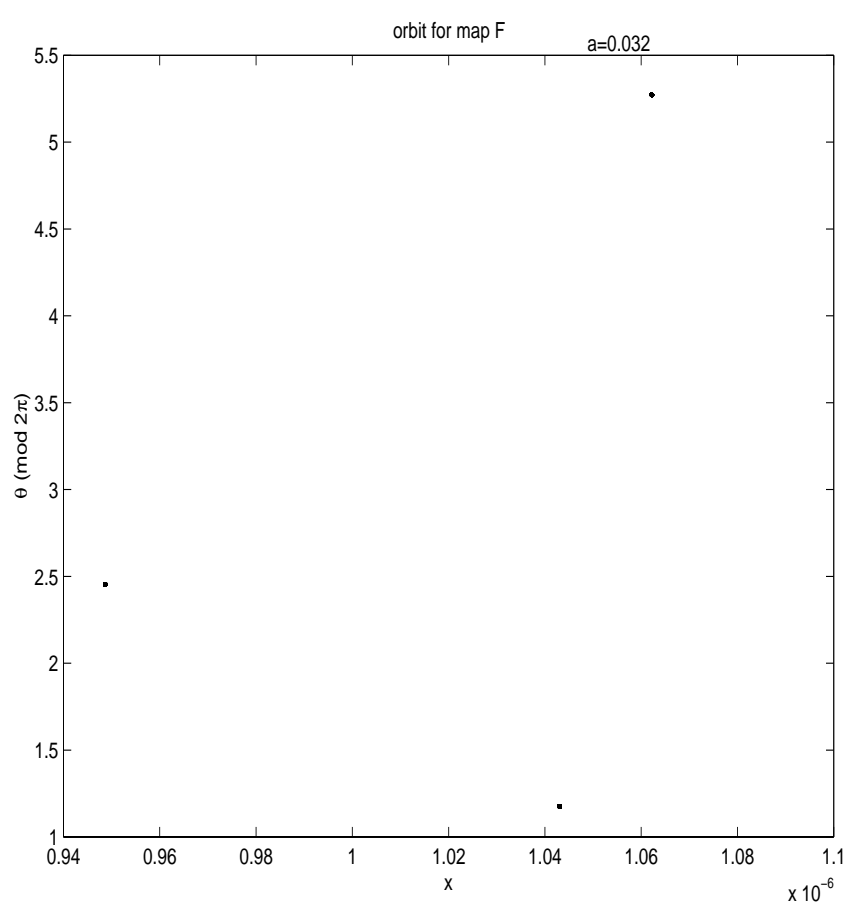

Fig. 8. Orbit of the map $F$ for $a=0.032$ for the last 3000 iterations.

orbits of $F$ for different values of parameter $a$ with $a=0.005,0.032,0.8$ in Figs. 7-9, respectively. For $a=0.005$ we obtain an invariant closed curve as the $\omega$-limit set. This explains why we have a triangular region for $a$ near 0 . When $a=0.032$, we obtain 


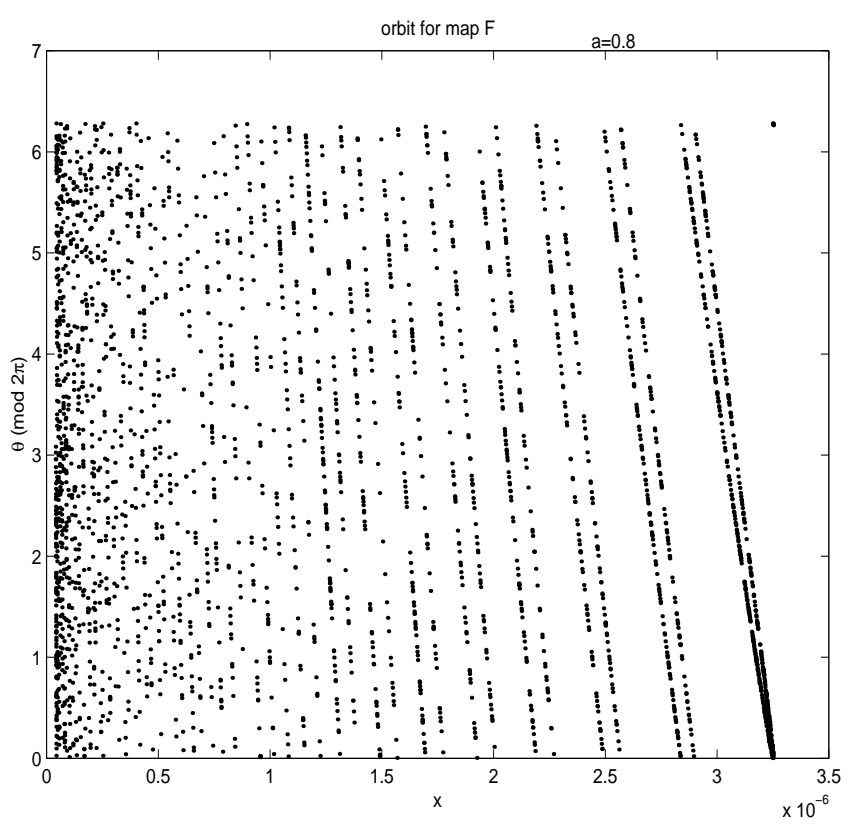

Fig. 9. Orbit of the map $F$ for $a=0.8$ for the last 3000 iterations.

period three orbit. For $a=0.8$, as we predict, the orbit is chaotic.

\section{Concluding Remarks}

We have shown that the behavior of the perturbed May-Leonard system depends intimately on the values of three parameters: $\nu=\nu_{11} \nu_{21} \nu_{31}, \eta=$ $\left(\left(1 / \lambda_{23}\right)+\left(\nu_{21} / \lambda_{13}\right)+\left(\nu_{11} \nu_{21} / \lambda_{33}\right)\right)$, and $a$. The parameters $\nu$ and $\eta$ are defined to be some combinations of eigenvalues of variational matrices at equilibrium points and reflect some relations between competing coefficients. The parameter $a$ is of the type of average amplitude of the external periodic forcing. In principle, it is possible to express it as some integral of the external force over the countour of hetroclinic orbits $O_{i}, i=1,2$,
3 , in the spirit of the Melnikov integral. However, this problem does not enter the scope of the paper. Anyway, it is clear now that being periodically perturbed, the system of three species, may behave periodically, quasiperiodially or chaotically, depending on the specific character of a perturbation.

\section{Acknowledgments}

V. Afraimovich would like to express his gratitude to the people at the National Center of Theoretical Science for their hospitality while he visited Taiwan in 1999.

\section{References}

Afraimovich, V. S., Bykov, V. V. \& Shil'nikov, L. P. [1983] "On structurally unstable attracting limit sets of Lorentz attractor type," Trans. Moscow Math. Soc. 44, 153-216.

Afraimovich, V. S., Gavrilov, N. N., Lukyanov, V. I. \& Shil'nikov, L. P. [1985] Main Bifurcation of Dynamical Systems (Gorky University Press, Gorky).

Afraimovich, V. S. \& Hsu, S. B. [1998] Lectures on Chaotic Dynamical Systems (National Tsing-Hua University, Hsinchu, Taiwan).

Burns, K. \& Weiss, H. [1995] "A geometric criterion for positive topological entropy," Commun. Math. Phys. 192, 95-118.

Chi, C. W., Hsu, S. B. \& Wu, L. I. [1998] "On the asymmetric May-Leonard model of three competing species," SIAM J. Appl. Math. 58, 211-226.

Katok, A. \& Hasselblatt, B. [1995] Introduction to the Modern Theory of Dynamical Systems (Cambridge University Press).

May, R. M. \& Leonard, W. J. [1975] "Nonlinear apects of competition between three species," SIAM J. Appl. Math. 29, 243-253.

Waltman, P. [1983] Competition Models in Population Biology (SIAM, PA). 